\title{
Klinisch relevante Ergebnisse vom SABCS 2006
}

\author{
Gesprächsleitung: Nadia Harbeck (München) und Raimund Jakesz (Wien) \\ Teilnehmer: Michael Gnant, Wien Wolfgang Janni, München Volker Möbus, Frankfurt/M. \\ Günther Steger, Wien Michael Stierer, Wien Christoph Thomssen, Halle/Saale
}

\section{Frage 1: Welche Patientinnen in der Prämenopause können adjuvant ausschließlich endokrin behandelt werden?}

Gnant: Generell können rezeptorpositive Patientinnen mit eindeutig hormonempfindlicher Brustkrebserkrankung und/ oder geringem Rezidivrisiko als adjuvante Therapie allein endokrin behandelt werden.

Janni: Jene Patientinnen, die bei denen aufgrund des niedrigen Rezidivrisikos der zusätzliche Therapievorteil durch eine adjuvante Chemotherapie unter 3-5\% liegt. Die Therapie sollte dann mit einer Kombination aus Gonadotropin Releasing Hormone (GnRH) und Tamoxifen erfolgen.

Möbus: Die ausschließliche endokrine Behandlung bei hormonrezeptorpositiven, prämenopausalen Patientinnen muss sicherlich auf ein prognostisch sehr günstiges Patientinnenkollektiv beschränkt bleiben. Hierzu gehören z.B. Patientinnen, die älter als 40 Jahre, N0, G1 und HER2-negativ sind und einen kleinen Primärtumor hatten. Aus randomisierten Studien wissen wir zwar, dass GnRH-Analoga bei prämenopausalen Patientinnen genauso effektiv sind wie die in diesen Studien verwendeten Chemotherapieregime, hierbei ist jedoch zu bedenken, dass es sich bei den eingesetzten Chemotherapieregimen ausschließlich um CMF (Cyclophosphamid, Methotrexat und 5-FU) oder inadäquat dosiertes FEC (5-FU, Epirubicin und Cyclophosphamid) gehandelt hat. $\mathrm{Ob}$ die alleinige Therapie mit GnRH-Analoga äquivalent ist $\mathrm{zu}$ den heute etablierten, deutlich wirkungsstärkeren Chemotherapieregimen, darf bezweifelt werden. Der Verzicht auf eine Chemotherapie in der Prämenopause sollte daher einem kleinen, prognostisch sehr günstigen Subkollektiv vorbehalten bleiben.
Steger: Jene Patientinnen, die entsprechend der Definitionen des St. Gallen-Consensus 2005 einen eindeutig hormonabhängigen Tumor haben und deren Generalisierungsrisiko als niedrig oder mittelgradig eingestuft werden kann, können adjuvant ausschließlich endokrin behandelt werden.

Stierer: Da die Datenlage sehr dürftig ist, scheint derzeit die Gabe einer ausschließlich endokrinen adjuvanten Therapie in der Prämenopause nur bei Patientinnen mit endokriner Empfindlichkeit (ER $>10 \%$ positive Zellen, $\mathrm{PgR}>10 \%$ positive Zellen) und niedrigem bis mittlerem Risiko nach den St. Gallener Richtlinien möglich. Eine wichtige Entscheidungshilfe könnten in Zukunft die Ergebnisse der laufenden PERCHE Studie liefern.

Thomssen: Entscheidend ist zunächst die Frage der Sensitivität der Tumoren gegenüber endokrinen Therapien. St. Gallen 2005 unterscheidet endokrin sensitive Tumoren von nicht sensitiven und solchen zweifelhafter endokriner Sensitivität. Eine rein endokrine Therapie ist grundsätzlich bei endokrin sensitiven Tumoren mit sehr niedrigem Rezidivrisiko möglich oder wenn nur ein geringer Effekt der zytostatischen adjuvanten Therapie zu erwarten wäre. Diese Kriterien sind bei prämenopausalen Frauen erfüllt bei Alter > 35 Jahre, N0, G1 oder G 2, Tumorgröße $<2 \mathrm{~cm}$, fehlender Gefäßinvasion und eindeutiger endokriner Sensitivität. Bei G1-Tumoren dürfte eine Tamoxifentherapie ausreichend sein, bei G2-Tumoren würde ich zusätzlich für 2 Jahre ein GnRH-Analogon geben. Für Tumoren mit HER2-Überexpression, negativem Progesteronrezeptorstatus und erhöhten Invasionsfaktoren uPA/ PAI-1 wird eine zweifelhafte endokrine Sensitivität angenommen, diese sollten adjuvant nicht ausschließlich endokrin behandelt werden.

\begin{tabular}{ll}
\hline KARGER & ๑ 2007 S. Karger GmbH, Freiburg \\
Fax +49 7614520714 & Accessible online at: \\
$\begin{array}{l}\text { E-mail Information@Karger.de } \\
\text { www.karger.com }\end{array}$ & www.karger.com/brc
\end{tabular}


Frage 2: Ist die dosisdichte Therapie außerhalb von Studien Ihrer Meinung nach Standard?

Gnant: Nein.

Janni: Nein, da der Gesamt-Überlebensvorteil begrenzt ist, und die jeweiligen Vergleichsarme in den beiden Studien nicht der effektivsten Therapie in der nicht-dosisdichten zytostatischen Behandlung entsprechen.

Möbus: Es gibt zwei große randomisierte Studien, welche die Überlegenheit einer dosisdichten Chemotherapie im Vergleich zu einer 3-wöchigen Chemotherapie eindeutig belegt haben. Hierzu gehören die CALGB-C9741-Studie und die ETC-Studie der AGO. Die retrospektive Subgruppenanalyse der AGO-Studie hat gezeigt, dass Patientinnen mit 10 und mehr befallenen Lymphknoten und einem medianen Followup von 5 Jahren einen signifikanten Überlebensvorteil im Vergleich zu einer konventionellen Chemotherapie haben. Auch Frauen mit 4-9 befallenen Lymphknoten profitieren eindeutig von der dosisdichten Chemotherapie, wenngleich zum jetzigen Zeitpunkt der Vorteil noch nicht signifikant ist. Bei der Indikation zu einer alleinigen antihormonellen Therapie würden wir diese unabhängig vom HER2-Status treffen. Von der CALGB-C9741-Studie liegt keine publizierte, retrospektive Subgruppenanalyse vor. Auch wir geben die dosisdichte Chemotherapie bevorzugt innerhalb von Studien (GAIN-Studie), würden aber auch Hochrisikopatientinnen mit 4 und mehr befallenen Lymphknoten, welche eine Therapie unter Studienbedingungen ablehnen, bevorzugt mit dem dosisdichten ETC-Protokoll behandeln.

Steger: Nein.

Stierer: Diese Therapieform stellt meines Erachtens für Hochrisikopatientinnen (z.B. Hormonrezeptor (HR)-Negativität, Ki67 erhöht, massiver Lymphknotenbefall) mit hohem Frühmetastasierungsrisiko eine diskutable Therapieoption dar, die im Tumormodell nach Gompertz (Norton-Simon Hypothese) auch eine theoretische Rationale hat. Die Einbeziehung dieser Therapieform in den Standard erscheint möglich und könnte im Rahmen der nächsten St. Gallen-Konferenz in die Guidelines aufgenommen werden. Es existieren schon zwei positive Studien (INT 9741, ETC-AGO Trial), in welchen Vorteile dieser Therapieform gegenüber konventionellen Therapieschemata gezeigt worden sind. Mit Spannung zu erwarten sind auch die Ergebnisse von NSABP B38 (6× Docetaxel, Doxorubicin, Cyclophosphamid (TAC) vs. dose-dense $4 \times$ Doxorubicin, Cyclophosphamid $($ AC) $\rightarrow$ 4× Paclitaxel (PAC) vs. dose-dense $4 \times \mathrm{AC} \rightarrow 4 \times \mathrm{PAC}+$ Gemcitabin).

Thomssen: Nach der derzeitigen Studienlage (CALGB \# 9741, AGO N4+) ist für Patientinnen mit nodalpositiven Hochrisiko-Tumoren ein eindeutiger Benefit durch dosis- dichte Therapien gezeigt worden. Dies bestätigt sich auch in der anlässlich SABCS 2006 vorgestellten ersten InterimAnalyse der MA.21 Studie. $\mathrm{FE}_{120} \mathrm{C} \times 6$ und dosisdicht $\mathrm{EC} \times 6$ sind effektiver als die Standardtherapie $\mathrm{AC} \rightarrow \mathrm{T}$. Grundsätzlich profitieren alle Patientinnen der Studien von der dosisdichten Therapie, in der Studie der CALGB sind es insbesondere die Patientinnen mit homonrezeptornegativen $\left(\mathrm{HR}^{-}\right)$ Tumoren. Bei diesen Patientinnen $\left(\mathrm{HR}^{-}\right)$und - aufgrund des Rezidivrisikos - auch bei Mammakarzinomen mit 4 oder mehr befallenen Lymphknoten würde ich eine dosisdichte Therapie außerhalb von Studien indiziert sehen.

\section{Frage 3: Ist der HER2-Status für Sie bei postmenopausalen rezeptorpositiven Patientinnen therapieentscheidend?}

Gnant: Ja, bei diesen Patientinnen würde ich Trastuzumab als Standardtherapie einsetzen, zusätzlich Chemotherapie für (fast) alle HER2-positiven Patientinnen mit invasivem Brustkrebs und als endokrine Therapie primär Aromatasehemmer.

Janni: Nein, auch nach der Trans-ATAC-Studie ist die Einschätzung, dass der HER2-Status entscheiden ist, nicht mehr zutreffend.

Möbus: Dies hängt natürlich partiell vom Alter der Patientin und den sonstigen Prognosefaktoren des Tumors ab. Bei mittlerem und hohem Risiko und einer Patientin in gutem Allgemeinzustand würden wir bei positivem HER2-Status sehr großzügig die Indikation zur Chemotherapie in Kombination mit Trastuzumab sowie gegebenenfalls einer zusätzlichen antihormonellen Therapie stellen.

Steger: Ja, der HER2-Status ist für uns therapieentscheidend.

Stierer: Im Falle eines HER2-positiven Mammakarzinoms erhält eine postmenopausale Patientin in jedem Fall als endokrine adjuvante Therapie wegen des hohen Frührezidivrisikos einen Aromatasehemmer «upfront». Wegen der fraglichen Hormonempfindlichkeit in dieser Situation ist eine sequentielle Chemo-Hormontherapie mit anschließender einjähriger Trastuzumabgabe angezeigt. Ebenso ist bei Vorliegen eines negativen Lymphknotenstatus eine intermediäre Risikosituation anzunehmen.

Thomssen: Die HER2-Überexpression eröffnet den effektiven Einsatz einer zielgerichteten Therapie mit Trastuzumab. Diese Möglichkeit sollte im Rahmen der geprüften und zugelassenen Indikationen auch bei postmenopausalen Patientinnen mit hormonrezeptorpositiven Tumoren und HER2-Überexpression genutzt werden: Patientinnen mit N0 $\geq 1 \mathrm{~cm}$ und N1 erhalten eine adjuvante Chemotherapie und Trastuzumab, 
solche mit $\mathrm{N} 0<1 \mathrm{~cm}$ und Patientinnen, die aufgrund ihrer Lebenserwartung und Komorbidität keine Chemotherapie erhalten sollten, sind davon ausgenommen. Für weitere Entscheidungen gibt es keine Evidenz, obwohl retrospektive Analysen und biologische Daten vermuten lassen, dass HER2-überexprimierende Tumoren besser auf Aromatasehemmer als auf Tamoxifen und besser auf Anthrazykline und Taxane als auf CMF reagieren.

Frage 4: Welche neuen prädiktiven oder prognostischen Tests haben Ihrer Meinung nach das größte Potential, die klinische Behandlungsentscheidung zu beeinflussen?

Gnant: Realistischerweise wird kurzfristig kein prognostischer Test die Behandlungsentscheidung beeinflussen können. Mittelfristig könnte der Agendia-Chip (MIND-ACT!) interessant werden.

Janni: Gene-Array Testung und Detektion von «Minimal Residual Disease» werden in der Zukunft sicher eine Rolle spielen.

Möbus: Hier sollte man eine konservative Einstellung vertreten. Wir haben in den letzten 20 Jahren gelernt, dass von den neu ausgerufenen, angeblich klinisch relevanten prädiktiven und prognostischen Markern sich kaum einer im Alltag etablieren konnte. So gab es z.B. hinsichtlich des klinischen Stellenwertes der Überexpression von Topoisomerase 2- $\alpha$ (TOP2A) in San Antonio eher enttäuschende Ergebnisse (BCIRG 006). Gleiches würde ich - prospektiv gedacht auch für das «Genetic Mapping» erwarten. Hingegen haben verschiedene Arbeiten in San Antonio noch einmal eindeutig den engen Zusammenhang zwischen der Höhe der Hormonrezeptorexpression und dem Ansprechen auf eine antihormonelle Therapie zeigen können.

Steger: Kurzfristig könnten möglicherweise der TOP2AStatus und der c-myc-Status zur weiteren Individualisierung der Chemotherapiewahl herangezogen werden und vielleicht auch der lösliche HER2-Rezeptor im Serum zur besseren Steuerung der Trastuzumabtherapie.

Stierer: Als ein sehr interessanter prädiktiver Faktor für das Ansprechen einer Anthrazyklintherapie hat sich das Vorliegen einer TOP2A-Überexpression erwiesen. Dabei zeigt sich, dass nur HER2 und TOP2A-überexprimierende Tumoren explizit von Anthrazyklinen profitieren; bei allen anderen wäre ein nicht-anthrazyklinhaltiges Schema ebenso geeignet (untersucht in BCIRG 006 und in NCIC CTG MA.5). Eine flächendeckende Verfügbarkeit ist aber noch nicht gegeben. Der beobachtete Zusammenhang zwischen spezifischer Anthrazyklinempfindlichkeit und HER2-Überexpression dürfte also in Wirklichkeit nur jene Untergruppe betreffen, die eine
TOP2A-Überexpression aufweisen. Kosten, fragliche Reproduzierbarkeit und Erhöhung des Arbeitspensums stehen derzeit der Einführung weiterer hoffnungsvoller Verfahren in die Klinik entgegen. Dies gilt z.B. für die Kombinationsanalyse von $\mathrm{ER} \alpha, \mathrm{PgR}$ und BRCA-1 mRNA zur Klärung der Frage der AI-Ansprechbarkeit. Zweifelsohne gehört diesen molekularbiologischen Tests mit einer völlig neuen Dimension mittelfristig die Zukunft. Die Präsenz disseminierter Tumorzellen im Knochenmark bzw. die Persistenz solcher Zellen nach adjuvanter Therapie stellen negative Prognosefaktoren dar. Inwiefern sie ihre klinische Bedeutung durch die Entwicklung standardisierter Genexpressionsanalysen verlieren werden, ist noch unklar, erscheint aber wahrscheinlich.

Thomssen: Unter den verfügbaren Instrumenten ermöglichen Grading, Alter und die Bestimmung der Invasionsfaktoren uPA und PAI-1 die am besten validierte Möglichkeit der Abschätzung des Rezidivrisikos. Die Prädiktion des Therapiebenefits erfolgt nach wie vor am besten mittels Hormonrezeptorstatus (für endokrine Therapien) und HER2-Status (für Trastuzumab). Genexpressonsprofile (als Microarrays oder Oncotype DX) sind vielversprechend, müssen aber durch die entsprechenden prospektiven Studien in den nächsten Jahren erst noch validiert werden. Für die Bestimmung der TOP2A-Expression haben sich die ursprünglichen Hinweise zur Vorhersage des Benefits anthrazyklinhaltiger Therapien in der aktuellen Auswertung der BCIRG-006 Studie nicht bestätigt. TOP2A ist somit keine therapieentscheidender Faktor und muss daher in der Routine nicht bestimmt werden.

\section{Frage 5: Welche Rolle spielt der Nachweis von Mikrometastasen in Sentinel-Lymphknoten bei der Behandlungsentscheidung?}

Gnant: Dies ist nach wie vor strittig. Wenn eine verlässliche Unterscheidung zwischen isolierten Tumorzellen (ITC) und Mikrometastasen möglich ist, dann ist die Entscheidung: Nachresektion versus keine, Nachbehandlung wie nodal-positiv versus nodal-negativ.

Janni: Wie Emiel Rutgers in seinem Vortrag treffend zusammengefasst hat: zunächst ist der Mikrometastasennachweis im Sentinel-Lymphknoten hauptsächlich für die Entscheidung zur Axilladissektion von Bedeutung. Die Therapieentscheidung sollte bei ansonsten negativer Axilla in der Regel davon nur wenig beeinflusst werden.

Möbus: Wenn sich in der endgültigen histologischen Aufarbeitung Mikrometastasen in einem Wächterlymphknoten nachweisen lassen, so erfolgt bei uns obligat die Indikation zu einer sekundären Axilladissektion. Da wir wissen, dass 
Patientinnen mit Mikrometastasen einen prognostisch ungünstigeren Verlauf haben, beeinflusst dies auch die Entscheidung zur Systemtherapie.

Steger: Bis dato wurde die reine Mikrometastasierung in Sentinel-Lymphknoten nicht als Lymphknoten-positiv mit den entsprechenden therapeutischen Konsequenzen gewertet. Die Daten aus dem NCI in Mailand, die von Alberti am SABCS 2006 präsentiert wurden, zeigen aber, dass dies möglicherweise zu hinterfragen ist. Prospektive Evaluierungen zu diesem Thema sind also dringend indiziert.

Stierer: Die prognostische Bedeutung von Mikrometastasen ist bis dato in 3 von 8 retrospektiven Untersuchungen gefunden worden, wobei diese die längsten Beobachtungszeiten aufweisen. Prospektive Untersuchungen von Cox und Giuliano mit kurzen Beobachtungszeiten zeigen keinen prognostischen Einfluss. Die noch offene prospektive Studie Z10 der ACOSOG könnte Klärung bringen. Eine rein auf Daten beruhende Therapieentscheidung ist derzeit nicht möglich. Praxisnah erscheint ein Algorithmus, welche eine durch H\&EFärbung entdeckte Mikrometastase dem N+-Status zuordnet. Eine lediglich durch Immunhistochemie detektierte Mikrometastase bei negativer Restaxilla kann somit der N0 Kategorie zugeordnet werden. Differenzierte adjuvante Therapieentscheidungen fallen letztlich je nach Platzierung des Tumors in die entsprechende Risikokategorie. Hier spielen eine Reihe von tumor- und auch patientenbezogenen Faktoren eine gewichtige Rolle, die die Bedeutung einer Mikrometastase in den Lymphknoten in dieser Situation stark relativiert.

Thomssen: Zu dieser Frage hat E. Rutgers, Amsterdam, auf dem SABCS 2006 klar Stellung genommen: In der Routine ist die immunhistochemische Fahndung nach Mikrometastasen nicht indiziert. Bei Nachweis von Lymphknotenmetastasen zwischen 0,2 $\mathrm{mm}$ und $2 \mathrm{~mm}$ Durchmesser im Sentinel-Lymphknoten würde eine Axilladissektion durchgeführt werden. Sind die weiteren axillären Lymphknoten frei von Tumorbefall, werden diese Patientinnen wie nodal negative angesehen, bei weiterem Tumorbefall würden diese Patientinnen als nodal positiv gelten. Sentinel-Lymphknotenbefall mit Tumorzellkomplexen von weniger als 0,2 $\mathrm{mm}$ Durchmesser werden generell als nodal negativ angesehen.

Frage 6: Welche Patientinnen benötigen lhrer Meinung nach keine adjuvante endokrine Behandlung über 5 Jahre hinaus?

Gnant: Patientinnen mit niedrigstem Risiko («Lowest Risk») und Osteoporosepatientinnen benötigen keine weitergehende endokrine Therapie.
Janni: Nodal negative Patientinnen mit insgesamt niedrigem Rezidivrisiko gehören zu dieser Gruppe.

Möbus: Ich nehme an, diese Frage beschränkt sich auf das Patientinnenkollektiv, das zum jetzigen Zeitpunkt bereits 5 Jahre Tamoxifen erhalten hat. Von den Daten der MA-17 Studie wissen wir, dass insbesondere die nodal positiven Patientinnen einen Vorteil von der erweiterten adjuvanten endokrinen Therapie haben. Dies gilt auch, wenn die mediane Therapiepause bereits 2,5 Jahre nach Beendigung der Tamoxifentherapie beträgt. Alle nodal positiven Frauen sollten motiviert werden, im Anschluss an die 5-jährige Tamoxifentherapie für weitere 5 Jahre Letrozol einzunehmen. Bei nodal positiven Frauen mit einer Reihe prognostisch ungünstiger Zusatzkriterien kann die verlängerte adjuvante endokrine Therapie ebenfalls in Betracht gezogen werden. $\mathrm{Zu}$ bemerken ist, dass diese positiven Daten trotz des vorzeitigen Abbruchs der Rekrutierung und der Entblindung durch die in San Antonio berichtete NSABP-B33 Studie bestätigt wurden.

Steger: Jene postmenopausalen Patientinnen, deren Generalisierungsrisiko als niedrig einzustufen ist, benötigen keine weitere endokrine Therapie.

Stierer: Da rezeptorpositive hormonempfindliche Mammakarzinome eine chronische Erkrankung mit Tendenz zur Spätmetastasierung darstellen, ist im Hinblick auf die vorliegende Datenlage die Therapieverlängerung über 5 Jahre hinaus sinnvoll. Bei Patientinnen über 80 Jahre, besonders bei negativem Lymphknotenstatus und Komorbidität, könnte ein Therapiestopp nach 5 Jahren erfolgen.

Thomssen: Nodal negativen Patientinnen würde man derzeit keine erweiterte adjuvante endokrine Therapie über 5 Jahre hinaus anbieten. Die bekannten Studien (MA.17 und ABCSG 6a) und auch die bei SABCS 2006 vorgestellten Daten zur erweiterten Therapie mit Exemestan haben keine neuen Aspekte zu dieser Frage erbracht.

\section{Frage 7: Gibt es Patientinnen, die bei DCIS oder invasivem Mammakarzinom nach brusterhaltender Therapie keine Strahlentherapie benötigen?}

Gnant: Patientinnen mit invasivem Mammakarzinom über 70 mit niedrigstem lokoregionalem Risiko: hochpositiver Rezeptorstatus, nodal negativ, Antihormontherapie, gut differenziert. Bei DCIS: wenn alle Resektionsränder über $10 \mathrm{~mm}$ liegen.

Janni: Derzeit eine ungeklärte und kontrovers diskutierte Frage. Vermutlich kann man auf eine Strahlentherapie am ehesten bei älteren Patientinnen über 70 Jahren mit kleinen Tumoren und prognostisch günstigen Befunden verzichten. 
Möbus: Auch wenn die vorliegenden randomisierten Studien zu keiner verbindlichen Definition von Subkollektiven geführt haben, die nicht von einer postoperativen Nachbestrahlung profitieren, erscheint es nicht wünschenswert im Sinne eines Automatismus - alle Patientinnen mit operiertem DCIS einer postoperativen Strahlentherapie zuzuführen. Dies gilt auch vor dem Hintergrund, dass mit dem Anlaufen des Mammographie-Screenings die Diagnosestellung eines DCIS deutlich zunehmen wird. Wenngleich die methodisch sehr sorgfältigen Arbeiten von Silverstein nicht randomisiert sind, glaube ich, dass bei einem DCIS $<1 \mathrm{~cm}$ und einem Sicherheitssaum von $1 \mathrm{~cm}$ im Gesunden durchaus von einer Strahlentherapie Abstand genommen werden kann.

Steger: Außerhalb von klinischen Studien sollte die adjuvante Strahlentherapie bei diesen Indikationen immer zum Einsatz kommen.

Stierer: Beim invasiven Mammakarzinom bei Patientinnen über 70 Jahre mit negativen Lymphknoten, positivem Hormonrezeptor und adjuvanter endokriner Therapie erscheint unter Berücksichtigung der vorliegenden Daten (ABCSG 8A, CALGB 9343) ein Verzicht auf die Radiotherapie möglich. Beim DCIS kann unter Berücksichtigung der vorliegenden Daten, einschließlich der Intergroup Studie E5194, bei G1,2-Läsionen unter $1 \mathrm{~cm}$, bei Schnittrandweiten zwischen 5 und $10 \mathrm{~mm}$ und einem Alter über 40 Jahren auf Radiotherapie nach «Informed Consent» verzichtet werden. Weitere Aufschlüsse sollten die noch ausständigen Resultate des TROG Trials 98.04 (Randomisierung zwischen Exzision +/- Radiotherapie +/- Tamoxifen) erbringen.

Thomssen: Die aktuell vorgestellte Studie der ECOG belegt, dass die Strahlentherapie nach brusterhaltender Operation (BCS) des DCIS die Zahl der Rezidive signifikant verringern kann. Dies ist auch gegenüber der Inzidenz kontralateraler Zweitkarzinome eindrucksvoll gezeigt worden. Schwächen dieser Studie sind offene Fragen, wie die Bewertung des tumorfreien Randes, der Lokalisation des Rezidives etc. Eine weitere Studie (Hughes K. für CALGB; RTOG; ECOG) hat gezeigt, dass ältere Patientinnen (>70 Jahre) mit kleineren hormonrezeptor-positiven Tumoren (T1N0) zumindest keinen Überlebensvorteil von der Bestrahlung nach BCS haben. Dies bestätigt ältere Daten von Veronesi, der ähnliches beobachtet hatte. Fazit: Ich sehe weiterhin bei DCIS $<2 \mathrm{~cm}$ mit ausreichend großen läsionsfreien Rändern keine Indikation zur Bestrahlung der Brust. Die Daten für die ältere Frau bei kleinen rezeptorpositiven Tumoren (T1N0) ermutigen dazu, auch bei diesen im Einzelfall auf die Radiotherapie der betroffenen Brust zu verzichten.
Frage 8: Abgesehen von Trastuzumab: Welchen Stellenwert haben Targeted Therapies bei der Behandlung des Mammakarzinoms? Gab es hierzu in San Antonio neue Erkenntnisse?

Gnant: Targeted Therapies sind die Zukunft. Bereits jetzt gibt es interessante Therapieansätze: Lapatinib nach Trastuzumabversagen, Bevacizumab vielleicht, Tyrosinkinaseinhibitoren eventuell auch in Kombination mit Aromataseinhibitoren.

Janni: Aus meiner Sicht gibt es hierzu keine wesentlichen neuen Erkenntnisse vom SABCS. Das größte Potential hat derzeit sicherlich Lapatinib, welches in zahlreichen neo-/ adjuvanten Studien untersucht wird.

Möbus: Tyrosinkinaseinhibitoren, insbesondere Lapatinib, und Anti-Angiogenesefaktoren haben bzw. werden mit Sicherheit Eingang in die metastasierte und auch in die adjuvante Therapie des Mammakarzinoms finden. Entsprechende Studien sind bereits initiiert. Der Einsatz von Lapatinib und von Bevacizumab in der metastasierten Situation stellt aufgrund der Datenlage schon heute eine Therapieoption dar. Trotz der neuen «Targeted Therapies» bin ich davon überzeugt, dass beim nodal positiven Mamakarzinom die Chemotherapie der Eckpfeiler der adjuvanten Therapie bleiben wird und um die Targeted Therapies zum Nutzen der Patientinnen ergänzt werden wird.

Steger: Bevacizumab und Lapatinib zeigen durchaus vielversprechende erste Ergebnisse in der Palliativtherapie und können meines Erachtens bereits jetzt im Einzelfall außerhalb von klinischen Studien zum Einsatz kommen. Relevante neue Ergebnisse hierzu wurden aber in San Antonio 2006 nicht präsentiert.

Stierer: Neben Trastuzumab kann Bevacizumab in Kombination mit Paclitaxel beim metastasierten Mammakarzinom erfolgreich als Erstlinientherapie alle wesentlichen Endpunkte im Vergleich zur Paclitaxelmonotherapie deutlich verbessern (K. Miller, ASCO 2005). Für Patientinnen mit Trastuzumabresistenz sollte in Zukunft mit dem oralen HER1- und HER2-Inhibitor Lapatinib eine Therapieoption offen stehen. Kombinationen mit Capecitabin haben jedenfalls zur Optimierung des Therapieeffektes im Vergleich zu Capecitabinmonotherapie bei metastasiertem Brustkrebs nach Anthrazyklin-, Taxan- und Trastuzumabversagen geführt (Cameron et al.). Erste Hinweise auf Ansprechen einer Lapatinibmonotherapie konnten im neoadjuvanten Setting gesehen werden (Christofanilli et al.). Ob Lapatinib das «bessere Trastuzumab» ist, wird im adjuvanten Setting zu prüfen sein. Zweifelsohne ist das günstige Nebenwirkungsprofil (weniger Kardiotoxizität als Trastuzumab, eher gastrointestinales Nebenwirkungsprofil) und die orale Verabreichungsform ein gewisser Vorteil. Im Rahmen der neuen Targeted Therapies stellen 
jedenfalls beim Mammakarzinom mit Lapatinib und Avastin zwei wichtige Ergänzungen im Behandlungsspektrum zur Verfügung, deren exakte Positionierung im therapeutischen Algorithmus noch unklar ist.

Thomssen: Untersuchungen von Transplantattumoren an der Nacktmaus weisen daraufhin (z.B. CK Osborne et al.), dass gerade die Kombination verschiedener Targeted Therapies das Wachstum von Mammakarzinomzellen nachhaltig unterdrücken kann. In ersten klinischen Daten (M Pegram et al.) wird die Wirksamkeit der Kombination Trastuzumab und Bevacizumab bestätigt. Die vorgestellten klinischen Daten konzentrieren sich im Übrigen auf die Nutzung einzelner Targeted Therapies mit Chemo- und Hormontherapie. Unverändert gilt, dass die Therapie mit dem VEGF-Antikörper Bevacizumab in Kombination mit Paclitaxel hochwirksam ist und effektiver als Paclitaxel alleine. Auch zur Kombination mit Docetaxel, Abraxane ${ }^{\circledR}$ und Capecitabin wurden vielversprechende Daten erarbeitet. Insbesondere konnte für das «Small Molecule» Lapatinib, welches mit der Tyrosinkinase von EGF-R und HER2 interagiert, sehr deutlich die hohe Wirksamkeit in Kombination mit Capecitabin bei HER2überexprimierenden Tumoren im Vergleich zu Capecitabin allein bestätigt werden (D Cameron et al.). Die Wirksamkeit von Lapatinib konnte in kleineren Studien auch für die Kombination mit Taxanen gezeigt werden und - im Labor - auch für die Kombination mit endokriner Therapie. Interessant ist auch die Möglichkeit, mittels Impfung gegen den HER2Rezeptor eine Remission zu erreichen.

\section{Frage 9: Was ist Ihrer Meinung nach die optimale adjuvante Therapie des HER2-positiven Mammakarzinoms?}

Gnant: Derzeit Chemotherapie und Trastuzumab für 1 Jahr.

Janni: Sequientielle Taxantherapie mit simultanem Trastuzumab in der Taxansequenz oder Docetaxel/Carboplatin/ Trastuzumab (TCH).

Möbus: Das ist sicherlich die Kombination von Chemotherapie und Antikörpertherapie. Ob eine simultane Chemo-Antikörpertherapie einer sequentiellen definitiv überlegen ist, kann zum jetzigen Zeitpunkt nicht beantwortet werden. Trotz der positiven Daten der BCIRG-006 Studie zur Kombination von Platin/Docetaxel/Trastuzumab handelt es sich erst um die zweite Interimsanalyse, so dass es noch zu früh ist, eine anthrazyklinfreie Chemotherapie zum Standard auszurufen. Der längere Follow-up wird die wichtige Frage entscheiden, ob im Kollektiv der HER2-überexprimierenden Patientinnen bei der Gabe von Trastuzumab auf eine anthrazyklinhaltige Chemotherapie verzichtet werden kann. Unbefriedigend ist, dass wir keine Daten zur Kombination einer dosisdichten Chemotherapie mit Trastuzumab haben, unabhängig davon, ob diese Therapie simultan oder sequentiell erfolgte.

Steger: Nach den vorliegenden Studienergebnissen der 4 groBen adjuvanten Studien muss allen Betroffenen die adjuvante Trastuzumabtherapie über $1 \mathrm{Jahr}$ angeboten werden und das entsprechende kardiologische Monitoring durchgeführt werden. Je nach Chemotherapieschema kann überlegt werden, Trastuzumab parallel zur Taxantherapie zu verabreichen. Eine eventuell mögliche Verkürzung der Therapiedauer entsprechend der FinHER-Daten muss erst propektiv geprüft werden. Auf Tamoxifen sollte bei HER2-positiven Tumoren derzeit verzichtet werden, eine alleinige adjuvante Therapie mit Aromatasehemmern und Trastuzumab bei postmenopausalen Patientinnen kann im Moment mangels entsprechender Daten nicht als Routinevorgehen angesehen werden. Bei notwendiger adjuvanter Strahlentherapie kann die Trastuzumabtherapie parallel geführt werden.

Stierer: Bei N0-Situation: 4× Docetaxel und Cyclophosphamid, synchron dazu für 1 Jahr Trastuzumab (3-wöchentlich) nach Abschluss der Chemotherapie, sequentiell bei hormonrezeptorpositiven Patientinnen Aromatasehemmer. Bei N+Situation: Anthrazyklin gefolgt von Taxan, synchroner Beginn von Trastuzumab für 1 Jahr (3-wöchentlich) mit Beginn der Taxantherapie, bei hormonrezeptorpositiven Patientinnen nach Beendigung der Chemotherapie Aromatasehemmer sequentiell.

Thomssen: HER2 überexprimierende Patientinnen (nodalpositive oder nodal-negative mit Tumoren $>1 \mathrm{~cm}$ ) sollten einer adjuvante Therapie mit Trastuzumab für ein Jahr erhalten. Grundsätzlich kann dies sequentiell oder simultan mit der laufenden Therapie erfolgen. Außerhalb klinischer Studien könnte - bei noch ungenügender Datenlage - die simultane Therapie mit Taxanen leichte Vorteile hinsichtlich Wirksamkeit haben. Standardtherapie wäre somit eine taxanhaltige adjuvante Chemotherapie mit einjähriger Trastuzumab-Therapie. Die endokrine Therapie richtet sich nach Rezeptorstatus und Menopausenstatus. Tamoxifen und Aromatasehemmer dürften jeweils wirksam sein. Aufgrund biologischer Überlegungen würde ich bei postmenopausalen Patientinnen wahrscheinlich dem Aromatasehemmer den Vorzug geben. Interessant ist die Frage der rein endokrinen Therapie mit Trastuzumab. Eine britische Studie (Mackay et al.) hat gezeigt, dass beim metastasierten Mammakarzinom die Kombination von Anastrozol mit Trastuzumab der alleinigen endokrinen Therapie überlegen ist (hinsichtlich Verlängerung des progressionsfreien Überlebens bei hormon-sensitiven und HER2-überexprimierenden Tumoren). Ob dies auf die adjuvante Therapie übertragen werden kann, ist ungeklärt. 


\section{Teilnehmer}

Univ. Prof. Dr. med. Michael Gnant

Präsident der Austrian Breast \& Colorectal Cancer Study Group

1090 Wien, Boltzmanngasse 24-26, Österreich

Tel +43 140-89230 Fax -90990

E-Mail michael.gnant@abcsg.at

PD Dr. med. Wolfgang Janni

Stlv. Klinikdirektor

I. Frauenklinik Innenstadt

Klinikum der Ludwig-Maximilians-Universität

Maistr. 11, 80337 München, Deutschland

Tel. +49 89 5160-4250/-4111, Fax -4186/-4662

E-Mail Wolfgang.Janni@med.uni-muenchen.de

Prof. Dr. med. Volker Möbus

Frauenklinik

Städtische Kliniken Frankfurt/M.-Höchst

Gotenstraße 6-8, 65929 Frankfurt/M., Deutschland

Telefon: +49 69300 599-45, Fax -46

E-Mail studien-frauenklinik@skfh.de

Univ. Prof. Dr. Günther Steger

Klinische Abteilung für Onkologie

Universitätsklinik für Innere Medizin I

Medizinische Universität Wien

1090 Wien, Österreich

Tel. +43 1 40400-5459, Fax -6081

E-Mail guenther.steger@meduniwien.ac.at

Univ. Prof. Dr. Michael Stierer

Abteilung für Chirurgie, Hanusch Krankenhaus

Heinrich-Collin-Straße 30, 1140 Wien, Österreich

Tel. +43 1 91021-2560, Fax -2654

E-Mail michael.stierer@wgkk.sozvers.at

Prof. Dr. med. Christoph Thomssen

Klinik und Poliklinik für Gynäkologie

Martin-Luther-Universität Halle-Wittenberg

Ernst-Grube-Strasse 40, 06097 Halle/Saale, Deutschland

Tel. +49 345 557-1847, Fax -1501

E-Mail christoph.thomssen@medizin.uni-halle.de 